\title{
Dynamic Action Semantics and Deontic Operators
}

\author{
Javier Gutiérrez-Rexach \\ The Ohio State University
}

\section{Introduction}

In this paper, I present a dynamic semantics of permission sentences which analyzes the effects of deontic operators in discourse. Permission statements are known to be problematic for logical and linguistic theories based on standard systems of modal logic. These systems lead to predictions that are contrary to what natural language intuitions dictate. The tension between traditional modai deontic semantics and the proper characterization of the meaning of permission statements involving the deontic expression may has surfaced in the form of a number of so-called paradoxes or problems. The most relevant ones in the philosophical literature are (i) the paradox of free choice permisssion (Von Wright, 1969; Kamp, 1973) and (ii) Lewis'(1979) problem about spurious permission. My proposal gives a solution to these two problems and to the intrincacies of the meaning of boolean connectives in statements of this sort.

\section{Free Choice Sentences}

Consider the following sentences:

(1) a. You may eat a banana or a pear

b. You may eat a banana

(2) a. You may go to San Francisco or stay in L.A.

b. You may go to San Francisco

Sentences (1a) and (2a) are "free choice" permission statements. If a speaker utters (1a), he is giving the addressee permission to eat either a banana or a pear. In other words, the addressee is free to choose from the options presented by the speaker: eat a banana, eat a pear or both. Therefore, when the speaker gives permission to the addressee to eat a banana or a pear, he is giving him permission to eat a banana. Our intuitions are, then, that (1a) entails (1b) and (2a) entails (2b). Nevertheless, this represents a problem for standard systems of deontic logic, as noticed by Ross (1941), Von Wright (1969) and Kamp $(1973,1979)$. In these systems, the entailment pattern that can be straigthforwardly derived is exactly the opposite, as the following proof shows:

(C) 1997 by Javier Gutiérrez-Rexach Aaron Lawson (ed), SALT VII, 180-196, Ithaca, NY: Cornell University. 
(3) $\vdash \mathrm{P} \phi \rightarrow \mathrm{P}(\phi \vee \psi)$

$\begin{array}{lll}\text { Proof: } & \mathrm{P}(\phi) & \text { Assumption } \\ & \mathrm{P}(\phi) \vee \mathrm{P}(\psi) & \vee \text { intro. } \\ & \mathrm{P}(\phi \vee \psi) & \text { Modal theorem } \\ & \mathrm{P}(\phi) \rightarrow \mathrm{P}(\phi \vee \psi) & \end{array}$

Kamp $(1973,1979)$ found the accounts presented to date not satisfactory and proposed a solution in the spirit of Lewis' (1979) proposal. The solution essentially consisted in spelling out the semantics of commands and permission statements introducing some concepts that in a certain respect anticipate the dynamic view of meaning. A command, according to Kamp and Lewis, restricts the options for action of the addressee. A permission statement broadens the options for action of the addressee. The options for action of an agent at time $t$ and world $w$ are formally defined as the possible continuations of $w$ after $t$ in which the agent fulfills all his obligations and forbears doing the things from which he is prohibited.

Let $\operatorname{Per}(w, t, B)$ deirote the set of possible continuations of $w$ after $t$ in which the agent $B$ fulfills his obligations and does not transgress anything he is prohibited from doing. Suppose that A utters in $w$ at $t$ sentence (4), and that $\mathrm{B}$ is the addressee of A's utterance.

\section{(4) Clean my table!}

Let $\mathrm{S}$ be the set of worlds in which B cleans A's table. Then, according to Kamp, the effect of A's command is to restrict the set of permitted continuations for $\mathrm{B}$ in $w$ at $t$ to those in which $\mathrm{B}$ cleans A's table:

(5) $\operatorname{Per}(w, t, B) \longmapsto \operatorname{Per}(w, t, B) \cap S$

A permission has the opposite effect in the set of options for action of a given agent. If a speal: r A tells B You may $\phi$ and $S^{\prime}$ is the set of worlds in which $\phi$ holds, then the effect of A's utterance is to enlarge the set of permitted options for B with $S^{\prime}$ :

(6) $\operatorname{Per}(w, t, B) \longmapsto \operatorname{Per}(w, t, B) \cup S^{\prime}$

In order to handle the entailment relation between $(1 \mathrm{a})$ and $(1 \mathrm{~b})$ or $(2 \mathrm{a})$ and (2b), Kamp (1973) introduces a new notion of entailment, P(ermission)entailment, defined as follows:

(7) $\phi$ P-entails $\psi$ iff in every situation the set of worlds added to the options of the addressee through the use of $\phi$ includes the set of worlds added to the set of options through the use of $\psi$

Kamp (1979) abandons this solution because he considered it problematic with respect to the spurious permisssion problem (Lewis' problem) that we will discuss below. In a nutshell, the above definition of entailment predicts that when an agent is granting the permission stated in (8a) he may also also be granting (8b). 
(8) a. You may go to San Francisco

b. You may go to San Francisco and burn my house

Sentence (8a) P-entails (8b) in all contexts in which if the proposition expressed by (9a) is true in a world $w$, then the proposition expressed by (9b) is also true in $w$. In other words, the set of worlds in which the addressee burns the speaker's house is a subset of the set of worlds in which the addressee goes to San Francisco.

(9) a. You burn my house

b. You go to San Francisco

The alternative solution proposed by Kamp (1979) is that the meaning of You may $\phi$ or $\psi$ is computed by separately calculating tie option space granted by You may $\phi$ and the option space granted by You may $\psi$ first, and combining the two of them by set-theoretic union. Then, writing $\llbracket \phi \rrbracket^{P e r}{ }_{w, t}$ to denote the set of worlds added to the options of the addr sse in $\langle w, t\rangle$ through the utterance of $\phi$, the following holds:

$\llbracket$ You may $\phi$ or $\psi \rrbracket^{P e r}{ }_{w, t}=$ $\llbracket$ You may $\phi \rrbracket^{P e r}{ }_{w, t} \cup \llbracket$ You may $\psi \rrbracket^{P e r}{ }_{w, t}$

This resolves the entailment problem but, as pointed out by Rohrbaugh (1995), it predicts the equivalence of (11a) and (11b):

(11) a. I permit you to eat an apple or a pear

b. I permit you to eat an apple or I permit you to eat a pear

The above sentences are not equivalent, nor are the following ones, illustrating the fact that VP-level disjunction does not have this same effect in permission sentences as sentence level (or speech-act level) disjunction does. VP-level disjunction under the scope of a permission operator amounts to a free choice permission statement, whereas the disjunction of two permission statements does not constitute a free choice statement but rather an exclusive permission statement: only one of the disjuncts is permitted.

(12) a. You may go to San Francisco or stay in L.A.

b. You may go to San Francisco or you may stay in L.A.

\section{Strong and Weak Readings}

Kamp (1979) also noticed that the sentence in (1a), repeated here as (13a), is ambiguous between two readings: a strong reading and a weak reading. 
In its "strong" or most salient reading it constitutes a free choice permission statement and entails (13b). In the "weak" reading, (13a) simply states the speaker's ignorance about which disjunct is actually permitted. In this reading, (13b) entails (13a).

(13) a. You may eat a banana or a pear

b. You may eat a banana

\subsection{Properties of the Strong Reading}

The strong reading of a permission sentence makes it a free choice permission statement: the speaker is granting permission to the addresse (14a) or telling the addressee that he is granting permission to a third person/s (14b):

(14) a. You may go to San Francisco or stay in L.A.

b. John may go to San Francisco or stay in L.A.

The strong reading of a permission statement may be paraphrased by a performative sentence:

(15) I hereby permit you to go to San Francisco or stay in L.A

(16) John may buy an Opel or a Honda =

I hereby permit John to buy an Opel or a Honda

A third characterizing property of the strong reading is that, as was discussed in the previous section, the following holds: $\mathrm{P}(\phi \vee \psi) \models \mathrm{P}(\phi)$. The opposite direction does not hold: $\mathrm{P}(\phi) \not \models \mathrm{P}(\phi \vee \psi)$. Also, in the strong reading the equivalence in (17) holds.

(17) $\mathrm{P}(\phi \vee \psi) \equiv \mathrm{P}(\phi) \wedge \mathrm{P}(\psi)$

The following example illustrates the above equivalence. If the speaker is giving permission to John to buy an Opel or a Honda by uttering (18a), then the permission granted by the speaker is the same as the permission granted when uttering $(18 \mathrm{~b})$.

(18) a. John may buy an Opel or a Honda

b. John may buy an Opel and John may buy a Honda

There is a variety of the strong reading in which the disjunction connective is construed as exclusive or. For instance, in the following discourse, the parent is most likely granting permission to buy a car or take a vacation but not both. 
(19) As a gift for your graduation, you may buy this expensive car or take the trip to the Bahamas that you wanted so much

\subsection{Properties of the Weak Reading}

A permission statement in its weak reading is a free choice permission report: it simply states the speaker's ignorance about which disjunct is actually permitted.

(20) John may buy an Opel or a Honda = John has been granted permission to buy an Opel or a Honda (but I don't know which one)

In the weak reading of the above sentence, the speaker is reporting to the addressee that a third person has granted John permission to buy an Opel or a Honda. Consequently, permission statements in their weak reading may not be paraphrased by a performative sentence.

In comparison to the strong reading, it can be observed that the reverse entailment patterns arise: $\mathrm{P}(\phi \vee \psi) \models \mathrm{P}(\phi)$ does not hold, but $\mathrm{P}(\phi) \models \mathrm{P}(\phi \vee \psi)$ holds. Another entailment pattern of interest is the following one, illustrated in $(21): \mathrm{P}(\phi) \vee \mathrm{P}(\psi) \models \mathrm{P}(\phi \vee \psi)$

(21) a. John may buy an Opel or John may buy a Honda $\models$

b. John may buy an Opel or a Honda

The weak reading of a permission sentence is a combination of a deontic and an epistemic statement. It cannot be considered a pure epistemic sentence. In other words, there is a subtle difference between the "permission report" reading and a pure epistemic reading. For instance, sentence $(21 \mathrm{~b})$ in its epistemic reading means that it is possible that John buy a Opel or a Honda - perhaps because he has not decided yet about which one, or the speaker does not know the content of his decision, or John is hoping to get a loan to finance the car, etc. These are all circumstances that make the epistemic reading true. The permission report reading requires something different and much more specific, namely that the speaker is reporting the effect of a deontic permission statement.

In some languages, the distinction between the strong and the weak reading is lexically realized - different adverbials are used - or is encoded by different complement types. In Spanish, a clause embedded under a modal verb has to be tenseless in the strong reading, whereas the weak or permission report reading requires subjunctive mood in the embedded clause. 
a. Puede Pedro ir al cine

may Pedro go to-the cinema

'Pedro may go to the cinema' (strong reading)

b. Pedro puede ir al cine

Pedro may go to-the cinema .

(23) Puede que Pedro vaya al cine

may that Pedro go-subj to-the cinema

'Pedro may go to the cinema' (weak reading)

\section{Actions in Dynamic Semantics}

The essence of the dynamic conception of semantics is to consider the basic meaning of a sentence to be not its truth-conditional content but its contextchange potential. The meaning of an arbitrary expression $\phi$ in a state $s$ is the change that $\phi$ brings about to $s$, or the update value of $\phi$. Let us assume that a conversation is in a discourse state $s$. Then, after processing a formula $\phi$, the discourse moves to a state $s^{\prime}$, as depicted in (24). The state $s^{\prime}$ is like $s$ except that those possibilities that are not compatible with what $\phi$ expresses (the condition $S$ ) are eliminated. Using a postfix notation, we write $s \llbracket \phi \rrbracket$ for the meaning of the formula $\phi$ in a state $s$, as in (25).

(25) $\quad s \llbracket \phi \rrbracket=s^{\prime}$ iff $s^{\prime} \subseteq s$ and $\mathrm{S}$

Information growth consists in the elimination of possibilities from an initial state $s$. When a formula $\phi$ is interpreted in a state $s$, some of the possibilities in $s$ are eliminated - those incompatible with $\phi-$, so the state $s^{\prime}$ resulting from the update of $s$ with $\phi$ is always a subset - not necessarily proper- of $s$.

Different branches of dynamic semantics vary with respect to what they consider to be an information state. In DPL (Groenendijk and Stokhof, 1991) and DMG (Groenendijk and Stokhof,1990) a state is a set of assignments of values to variables. In DRT, a state corresponds to a Discourse Representation Structure K, such that $K$ is the DRS built after processing a discourse (a finite sequence of sentences). In Dynamic Modal Logic a state is a set of worlds. This is the conception of a state that we will be adopting here. Furthermore, states will be epistemically construed, ie. we will be talking about the knowledge state of an agent rather than of a discourse state or a conversation state. This point is important in the type of account that will be devcloped, because: the dynamic content of permission statements will be taken to be its update 
potential with respect to the knowledge state of an agent (the speaker), not with respect to the deontic space of the addressee.

Dynamic action semantics adds to standard dynamic semantics a more refined analysis of action expressions. This analysis, I claim, is needed in order to give a correct account of the semantics of permission sentences. I present an extension of current dynamic modal frameworks (Veltman, 1996; Groenendijk, Stokhof and Veltman, 1995; Van Eijck and Cepparello, 1995) that incorporates a dy'namic semantics for actions, along the lines proposed in Pratt's (1978) process semantics, Van der Meyden's (1996) logic of permission and Hamblin's (1987) analysis of imperatives.

'The language of Dy'namic Action Logic (DAL) has two sorts of expressions: propositional or state expressions and action expressions. Propositions hold of states of affairs, whereas actions produce a change of state.

An action expression $\alpha$ is conceived of as denoting a program, ie. a set of sequences of states. Consider the following action expression: ${ }^{1}$

\section{(26) Kill(Caesar)(Brutus)}

Let us assume that the expression above denotes in a model $M$ a three membered set of sequences of states. Each sequence represents an execution of the action (Israel, Perry and Tutiya, 1993), i.e. the way of performing the action that results in that sequence of states.

$$
\llbracket \text { Kill(Caesat) (Brutus) }]_{M}=\left\{<s_{21} \ldots s_{3 \omega}\right\rangle,\left\langle s_{5} ; \ldots s_{6 n}\right\rangle,\left\langle s_{8_{1}} \ldots s_{30}\right\}
$$

In the process semantics given in (27), each sequence represents a different execution of Brutus' action of killing Caesar in $M$. For instance, in one execution he stabs Caesar three times, in another he stabs Caesar thirty times, and in the third one he stabs him fifteen times. An execution of an action is a transition between states. The minimal requirement that all the sequences in the denotation of $\alpha$ have to satisfy is that in the initial state of the sequence Caesar is not dead, and in the final one he is.

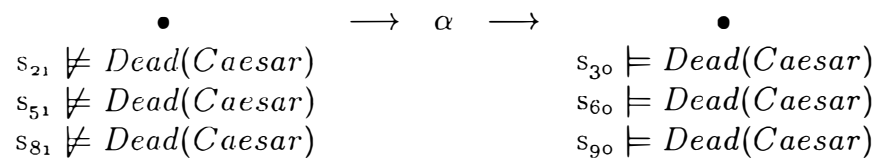

The syntax of the language of DAL is defined as follows: Let Act $=\{$ $\alpha, \beta, \gamma, \ldots\}$ be a set of atomic action constants and Prop $=\{\phi, \psi, \ldots\}$ a set of atomic propositions or state constants. The set of action expressions is defined to be the smallest set $\mathbf{A}$ containing Act such that if $\alpha, \beta \in \mathbf{A}$, then $\alpha \cup \beta \in \mathbf{A}, \alpha ; \beta \in \mathbf{A}$ and $\neg \alpha \in \mathbf{A}$. The set of propositional expressions of the language of DAL is the smallest set $\mathrm{P}$ containing Prop and satisfying:

1. If $\dot{\phi}, \psi \in \mathbf{P}$, then $-\dot{\sigma}, \phi \wedge \psi, \phi \vee \psi \in \mathbf{P}$. 
2. If $\alpha \in \mathbf{A}$, and $\phi \in \mathbf{P}$, then $\alpha \rightarrow \phi \in \mathbf{A}$.

3. If $\alpha \in \mathbf{A}$, then $\mho_{\alpha}, \square_{\alpha}, \pi \alpha \in \mathbf{P}$.

A model for the language of DAL is a tuple $M=\langle W, S, P, \tau, V\rangle$. W is a set of worlds and $W=\mathcal{P}(\mathcal{A})$, where $\mathcal{A}$ is a set of finitely many atomic sentences. This gives us the desired epistemic interpretation of worlds. $\Lambda$ world is a set of facts - atomic sentences - in the knowledge base of an agent (Veltman, 1996). $S \subseteq \mathcal{P}(W)$ is the set of states, so a state is a set of possible worlds. The knowledge state of an agent is, then, a family of sets of facts, i.e. those that constitute possible epistemic alternatives. Information growth is represented as elimination of some of those possibilities.

The relation $P$ is a relation between states, $P \subseteq S \times S$, where $<s_{i}, s_{j}>\in P$ iff the transition from state $s_{i}$ to state $s_{j}$ represents a permitted state transition. Generalizing from binary to n-ary sequences of states, we say that a sequence of states $\sigma=\left\langle s_{1} \ldots s_{n}\right\rangle$ is permitted, wtitten $\operatorname{Perm}(\sigma)$, iff every binary state transition in the sequence is in P. For instance, assume that $s_{21} \llbracket$ Get a payraise $($ John $) \rrbracket_{M}=\left\{\left\langle s_{21} \ldots s_{30}\right\rangle,\left\langle s_{21}, s_{51} \ldots s_{60}\right\rangle\right\}$ and in $s_{27}$ the proposition that John manipulates his sales report is true. Assuming that we are dealing with agents with standard ethical criteria, the first execution of the action is not considered to be permitted because it contains a state transition $\left\langle s_{26}, s_{27}\right\rangle$ which is not permitted: thus, $\left\langle s_{26}, s_{27}\right\rangle \notin \mathrm{P}$ and, as a consequence, $\neg \operatorname{Perm}\left(<s_{21} \ldots s_{30}>\right)$. This corresponds to the intuition that an execution of the action of getting a pay raise involving a manipulation of a sales report is not permitted, even if the rest of the transitions that bring about the completion of the action are permitted. Only the execution $\left\langle s_{21}, s_{51} \ldots s_{60}\right\rangle$ may be permitted, if all the transitions between states in the sequence are in the relation $\mathrm{P}$ of $M$.

The function $\tau: \mathbf{A} \rightarrow \mathcal{P}\left(S^{+}\right)$is the interpretation function for atomic action expressions $\alpha \in \mathbf{A}$, i.e. $\tau(\alpha)$ is the set of sequences of states denoted by $\alpha$. Finally, the function $\mathrm{V}$ maps atomic propositional constants $\phi$ to the set of worlds where the proposition holds.

The notions of support or acceptance of a propositional expression or formula in a state and the derived notions of dynamic entailment and equivalence between formulas are as follows: a state $s$ supports or accepts a formula $\phi$ iff updating $s$ with $\phi$ returns $s$; a formula $\psi$ is entailed by $\phi_{1} \ldots \phi_{n}$ iff the update of $s$ with $\phi_{1} \ldots \phi_{n}$ supports $\psi$; finally, two formulas are equivalent iff they entail each other.

(29) Support/Acceptance: $s \Vdash \phi$ iff $s \llbracket \phi \rrbracket=s$

Dynamic entailment: $\phi_{1} \ldots \phi_{n} \models \psi$ iff $\forall s .\left(\left(s \llbracket \phi_{1} \rrbracket\right) \ldots \llbracket \phi_{n} \rrbracket\right) \Vdash \psi$

Equivalence: $\phi \equiv \psi$ iff $\phi \models \psi \& \psi^{\prime}, \models \phi$

The expressions of the language of DAL are interpreted in a state $s$ as follows: 


\section{Semantics:}

1. $s \llbracket \phi \rrbracket=\{w \in s \mid w \in V(\phi)\}$

2. $s \llbracket \neg \phi \rrbracket=\{w \in s \mid w \notin V(\phi)\}$

3. $s \llbracket \phi \wedge \psi \rrbracket=(s \llbracket \phi \rrbracket) \llbracket \psi \rrbracket$

4. $s \llbracket \phi \vee \psi \rrbracket=\{w \in s \mid w \in V(\phi)$ or $w \in(s \llbracket \neg \phi \rrbracket) \llbracket \psi \rrbracket\}$

5. $s \llbracket \alpha \rrbracket=\{\sigma \mid \sigma \in \tau(\alpha) \& \operatorname{first}(\sigma)=s\}$

(where if $\sigma=\left\langle s_{1} \ldots s_{n}\right\rangle$, first $(\sigma)=s_{1}$ and $\operatorname{last}(\sigma)=s_{n}$ )

6. $s \llbracket \neg \alpha \rrbracket=\{\sigma \mid \sigma \notin \tau(\alpha) \&$ first $(\sigma)=s\}$

7. $s \llbracket \alpha \cup \beta \rrbracket=s \llbracket \alpha \rrbracket \cup s \llbracket \beta \rrbracket$

8. $s \llbracket \alpha ; \beta \rrbracket=\left\{\sigma_{1} \frown \sigma_{2} \mid \sigma_{1} \in \tau(\alpha) \& \sigma_{2} \in \tau(\beta) \& \operatorname{first}\left(\sigma_{1}\right)=s \& \operatorname{first}\left(\sigma_{2}\right)=\right.$ last $\left.\left(\sigma_{1}\right)\right\}$

9. $s \llbracket \alpha \rightarrow \phi \rrbracket=s$ iff $\forall \sigma \in s \llbracket \alpha \rrbracket[$ last $(\sigma) \Vdash \phi]$

10. Weak permission:

$s \llbracket \diamond \alpha \rrbracket=s$ iff $\exists \sigma \in s \llbracket \alpha \rrbracket$ such that $\operatorname{Perm}(\sigma)$

11. $s \llbracket \square \alpha \rrbracket=s \llbracket \neg \diamond \neg \alpha \rrbracket$

12. Strong permission:

$s \llbracket \pi \alpha \rrbracket=s$ iff $\forall \sigma \in s \llbracket \alpha \rrbracket$. Perm $(\sigma)$

Let us explain the clauses of the definition in more detail. The dynamic semantic value of a propositional expression $\phi$ in a state $s$ is the set of those worlds in $s$ in which $\phi$ holds. Similarly, if $s$ is updated with $\neg \phi$, the resulting state $s^{\prime}$ is the subset of $s$ constituted by the worlds in which $\phi$ does not hold. Another way of expressing this is the following:

$s \llbracket \phi \rrbracket=s-\{w \in s \mid w \notin V(\phi)\}$

$s \llbracket \neg \phi \rrbracket=s-\{w \in s \mid w \in V(\phi)\}$.

The dynamic content of the expression $\phi \wedge \psi$ is computed by updating first the state $s$ with $\phi$ and, as a result, climinating from $s$ the worlds that are not in $V(\phi)$. Then, the resulting state is updated with $\psi$, yielding a final state in which the worlds that are not in $V(\phi)$ and the worlds that are not in $V(\psi)$ are eliminated. The interpretation of dynamic disjunction has an exclusive flavor built in. Updating $s$ with $\phi \vee \psi$ restricts $s$ to the set of worlds that are either in $V(\phi)$ or are not in $V(\phi)$ but are in $V(\psi)$.

Action expressions do not have the same semantic type as propositional expressions. The update value of an action expression $\alpha$ in a state $s$ is not a state but a set of sequences of states. The dynamic content of an action expression $\alpha$ in a state $s$ is the set of sequences of states $\sigma$ in the denotation of $\alpha, \tau(\alpha)$, such that the first coordinate of $\sigma$ is $s$. Obviously, this represents the "atemporal" value of actions, which is essentially forward-looking and suffices for our purposes. The effect of the past and future operators would be captured as follows - where $\prec_{t}$ is an ordering relation between states: ${ }^{2}$ 


$$
\begin{aligned}
& s \llbracket P(\alpha) \rrbracket=\left\{\sigma \mid \sigma \in \tau(\alpha) \& \exists s^{\prime}\left[s^{\prime} \prec{ }_{t} s \& \text { last }(\sigma)=s^{\prime}\right]\right\} \\
& s \llbracket F(\alpha) \rrbracket=\left\{\sigma \mid \sigma \in \tau(\alpha) \& \exists s^{\prime}\left[s \prec{ }_{t} s^{\prime} \& \operatorname{Sirst}(\sigma)=s^{\prime}\right]\right\}
\end{aligned}
$$

The clauses defining operations on actions are straightforward. Updating $s$ with $\neg \alpha$ yields the set of sequences of states that are not in the denotation of $\alpha$ and such that their initial state is $s$. The dynamic conjunction of two actions $\alpha$ and $\beta$ is the pairwise concatenation of the sequences of states in $\tau(\alpha)$ and $\tau(\beta)$ satisfying the condition that the last state in a sequence $\sigma$ in $\tau(\alpha)$ and the first state in a sequence $\sigma^{\prime}$ in $\tau(\beta)$ are equal. The formula $\alpha \rightarrow \phi$ may be read as "if $\alpha$ then $\phi$ " or, perhaps more properly, "after $\alpha, \phi$ ". The proposition $\alpha \rightarrow \phi$ is supported in a state $s$ iff all the executions in the denotation of $\alpha$ with initial state $s$ are such that its last state supports $\phi$.

The effect of the modal operators $\diamond$ and $\pi$ corresponds to existential and universal quantification over sequences of states in the dynamic content of an action respectively. Thus, for any action $\alpha$ and state $s, \nabla_{\alpha}$ is supported by $s$ iff there is a permitted sequence in the denotation of $\alpha$ with initial state $s$ or, in other words, if some execution of $\alpha$ with initial state $s$ is permitted. Conversely, $s$ supports $\pi \alpha$ iff all executions of $\alpha$ with initial state $s$ are permitted.

\section{Explaining the strong/weak contrast}

The strong and weak readings of permission sentences are represented by the presence of the strong $(\pi)$ or weak $(\diamond)$ permission operator respectively. The strong operator models free choice, whereas the weak operator models partial ignorance about permission. The update of a state $s$ with $\pi \alpha$ adds the information that all the executions of $\alpha$ are permitted in $s$. In other words, all possible executions of the action $\alpha$ are considered as permitted by the agent whose knowledge state has been updated with $\pi \alpha$. Consider one of the examples presented previously:

(30) You may take a banana or an apple

By uttering sentence (30), the speaker is expressing a fact that holds in his knowledge base in a state $s$ or he is updating it with a fact that is supported by $s$. 'The strong reading of (30) states that any course of action in which the addressee takes a banana or an apple and such that it does not violate what the speaker considers permissible is permitted: all the executions $\sigma$ of the action of taking a banana or the action of taking an apple performed by the addressee are such that $\operatorname{Perm}(\sigma)$. This is precisely represented as follows:

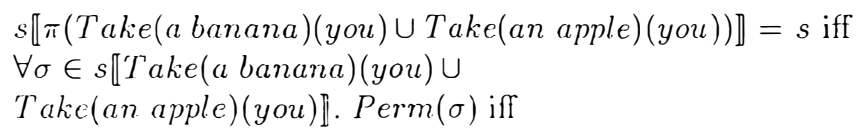




$$
\begin{aligned}
& \forall \sigma \in s \llbracket T a k e(\text { a banana })(y o u) \rrbracket \cup \\
& s \llbracket T a k e(\text { an appl } \epsilon)(\text { you }) \rrbracket . P \operatorname{Perm}(\sigma)
\end{aligned}
$$

A knowledge state $s$ supports $\pi$ (Take(a banana)(you) $\cup T a k e($ an apple $)(y o u))$ if and only if all the sequences $\sigma$ in the denotation of Take(a banana)(you) with initial state $s$ and all the sequences $\sigma^{\prime}$ in the denotation of Take(an apple) (you) with initial state $s$ are such that $\operatorname{Perm}(\sigma)$ and $\operatorname{Perm}\left(\sigma^{\prime}\right)$ holds. As is apparent, this says nothing about the knowledge state of the addressee, so here we depart from Kamp's conception of the meaning of a free permission statement like (30) as enlarging the option space of the addressee. The primary content of (30) is not to affect the epistemic or deontic state of the addresse, but rather to update the knowledge state of an agent --the speaker - with the assertion that the actions expressed in (30) are permitted according to the speaker. A different perspective would be to consider the effect of this permission statement on the agent it is addressed to. This would require ancillary notions such as satisfaction of or compliance with (30), that would demand a multi-agent semantics relating states in the knowledge bases of two (or more) different agents, in this case the speaker and the addressee. Interestingly, a multi-agent semantics is not needed to account for the data under consideration in this paper.

The following facts are immediately derived applying the definitions:

Fact 1: $\pi(\alpha \cup \beta) \models \pi(\alpha)$

Fact 2: $\pi(\alpha \cup \beta) \equiv \pi(\alpha) \wedge \pi(\beta)$

Fact 1 captures in a straithforward way the entailment pattern of strong readings, whereas fact 2 derives the equivalence pointed out in (15). The reading of (19) and (32) with exclusive or requires an additional binary operation on actions $(\sqcup)$ :

(32) You may buy a Porsche or a Corvette

The operation $\sqcup$ is defined as follows:

$s \llbracket \alpha \sqcup \beta \rrbracket=s \llbracket \alpha \rrbracket \cup s \llbracket \neg \alpha ; \beta \rrbracket$

The action $\alpha \sqcup \beta$ denotes in a state $s$ the union of the set of sequences in $\tau(\alpha)$ with first state $s$ and set of sequences in $\neg \alpha ; \beta$. Obviously, $\alpha ; \beta$ is not in the set of executions of $\alpha \sqcup \beta$ in $s$. The equivalence between the exclusive reading of (32) and sentence (33)

(33) You may buy a Porsche or you may buy a Corvette

is predicted by the following fact: 
Fact 3: $\pi(\alpha \sqcup \beta) \equiv \pi(\alpha) \vee \pi(\beta)$

Let us now consider the weak reading of permission sentences. A knowledge state $s$ supports $\nabla_{\alpha}$ iff there is at lcast an execution of $\alpha$ that is considered to be permitted in $s$. When a speaker utters (34), he is asserting that there is a course of action in which the addressee takes a banana or an apple and such that it does not violate what is permissible within the speaker's knowlege, as shown in (35).

(34) You may take a banana or an apple

(35) $s \llbracket \diamond(T a k e($ a banana $)(y o u) \cup$ Take $($ an apple $)(y o u)) \rrbracket=s$ iff $\exists \sigma \in s \llbracket$ Take $($ a banana $)($ you $) \cup$

Take(an apple)(you)』 such that $\operatorname{Perm}(\sigma)$, iff

$\exists \sigma \in s \llbracket T a k e($ a banana $)(y o u) \rrbracket \cup$

$s \llbracket$ Take (an apple) $(y o u) \rrbracket$ such that $\operatorname{Perm}(\sigma)$

The strong reading of a permission sentence is represented as universal quantification over sequences or executions because it does not seem plausible that if an agent is giving permission to do something and there is an execution $\sigma$ such that all the state transitions in $\sigma$ are in the permission relation $P$ - the set of permitted binary transitions-, it might still be the case that the execution $\sigma$ is not permitted. Our intuitions are that in the latter case the relevant agent is not giving a full or unrestricted permission or, in other words, blocking $\sigma$ as not permitted is inconsistent with all the state transitions involved being in $P$ and all other executions of the action being permitted. This explains why universal quantification over sequences captures strong permission.

On the other hand, the permission report reading just represents the idea that the speaker is aware that some execution of an action is permitted because he has been told so. The information that is available to him is that the execution under consideration is permitted. Thus, the operator representing the weak reading of the modal verb introduces existential quantification over sequences of states (executions) and not universal quantification.

This characterization of the weak reading allows for an interesting distinction: it may be the case that the speaker is reporting an action as permitted according to criteria that are not his own. In other words, it is possible that the speaker reports a permission that contradicts what his own criteria of permissibility are. The preferred reading of (36) is the one in which the speaker agrees or at least is not in open disagrecment with the permission he is reporting. Sentence (37) is more neutral in that respect.

(36) You may read that book.

(37) You are allowed to read that book. 
In Spanish, sentence (38), the correlate of (36), would be infelicitous if uttered by an agent who does not agrec with the permission he is reporting. In this case only sentence (39), the correlate of (37) abovc, would be felicitous.

(38) Puedes leer ese libro may-you read that book

$$
\text { Se te permite leer ese libro }
$$

IMPERS. you allows read that book

The proper treatment of this distinction would require models in which instead of a unique permissibility relation $P$, there is a family of such relations $P_{i} \subseteq S \times S$, where $i$ is an agent index. Then, the weak reading of (38) would be supported in a knowledge state $s$ of an agent $i$ uttering the sentence iff there is an execution of the action of reading a book such that all state transitions in that execution are in $P_{j}$, for $j$ an agent different from $i$, and are also in $P_{i}$. The weak reading of sentence (39) would be supported in a knowledge state $s$ of an agent $i$ iff there is an execution of the action of reading a certain book such that all state transitions in that execution are in $P_{j}$, for $j$ an agent different from $i$, and are not in $P_{i}$.

Facts 4 and 5 are again immediately derived by applying the definitions, and predict the properties described in section 3.2 above.

Fact 4: $\diamond(\alpha) \vee \diamond(\beta) \models \diamond(\alpha \cup \beta)$

Fact 5: $\diamond(\alpha) \models \diamond(\alpha) \vee \diamond(\beta)$

From fact 4, the entailment pattern in examplc (21), repeated here as (40), follows.

(40) a. John may buy an Opel or John may buy a Honda

b. John may buy an Opel or a Honda

When a speaker reports that John has been allowed to buy an Opel or John has been allowed to buy a Honda, as in (40a), the disjunctive connective is interpreted as exclusive: the speaker reports that John has been granted permission to buy either an Opel or a Honda, but not both. The sentence has only one weak reading, the exclusive one. The report reading in which or is interpreted as inclusive is not possible, so the translation of (40a) to the language of DAL captures its exact meaning.

(41) $\diamond($ Buy $($ an Opel $)($ John $)) \vee \diamond(B u y($ a Honda $)($ John $))$

Formula (41) entails (42): if a state $s$ supports (41) it also supports (42).

$$
\diamond(\text { Buy }(\text { an Opel })(\text { John }) \cup B u y(\text { a Honda })(\text { John }))
$$


The reverse direction does not hold. Why is this so? Because (42) is supported or accepted in a state $s$ in which John has been given permission to buy an Opel, a Honda or both. Formula (41) would not be accepted in $s$. This property of (42) mirrors the ambiguity of sentence (40b) which, in contrast to (40a), has two weak readings: one in which or is construed as exclusive and a second one in which or is construed as inclusive. ${ }^{3}$

Lewis' (1979) problem about permission does not arise in dynamic action semantics, because permission sentences do not merely enlarge the option set of the addressee. Only sequences of states consisting of permitted transitions are in the denotation of the permission operators. Therefore, from (43a) in its strong reading one cannot infer $(43 \mathrm{~b})$ because presumably most of the executions of the action burn my house are not permitted even if the two conjuncts are true in the same worlds.

(43) a. You may go to San Francisco

b. You may go to San Francisco and burn my house

If $(43 a)$ is understood in its weak reading and the speaker is reporting that somebody has allowed the addressee to go to San Francisco, the inference is valid in DAL. This case is not an instance of spurious permisssion because, as was discussed above, permission reports do not imply agreement with the permissibility of what has been granted.

\section{Extensions of the analysis}

\subsection{Decreasingness}

Rohrbaugh (1995) observes that permission sentences are decreasing in the internal argument of the verb: (44a) entails (44b).

(44) a. You may eat three apples

b. You may eat two apples

The decreasingness effect is predicted as a result of the presence of the permission operator and the execution-based sequence semantics for actions. Wc say that an action $\beta$ is an extension of or encompasses an action $\alpha(\alpha \leq \beta)$ iff $\tau(\alpha)=\left\{\sigma_{i} \mid \exists \sigma_{j} \in \tau(\beta)\right.$ such that $\sigma_{i}$ is a subsequence of $\left.\sigma_{j}\right\}$. Then, from the definition of the extension relation between actions and the semantics of the strong permission operator the following fact is derived:

Fact 6: $\alpha \leq \beta \wedge \pi \beta \models \pi \alpha$

This fact captures the intended inference in (41), but would erroneously predict that in (45) below the instructor. when allowing the student to writc 
two essays instead of taking the midterm, is also granting permission to write one essay instead of taking the midterm.

(45) Instead of taking the midterm you may write two essays

In the most prominent reading of (45) the decreasingness effect is blocked. This reading can be called a "package deal" reading: the addressee is granted permission to perform an action in which he writes two essays instead of taking the midterm. This effect blocks the decrasingness property and may be taken as pragmatically conditioned. ${ }^{4}$

\subsection{Permission in conditionals}

Consider sentence (46a):

(46) a. If you commit a traffic violation, then you may appeal it in court.

b. You may commit a traffic violation and appeal it in court.

When a permission operator occurs in the consequent of a conditional, like in (46a), the operator may not be exported out of the consequent. In other words, (46b) is not entailed by (46a). Some analyses of deontic sentences (Meyer, 1988) validate the inference from (46a) to (46b). This is so because the existence of a set of permitted states of affairs is assumed and actions are taken to be permitted when they result in a permitted state. The advantage of a process semantics for actions, such as the one proposed for DAL, in which state transitions rather than states are considered to be permitted or not permitted, is that $\alpha \rightarrow \pi \beta$ does not entail $\pi(\alpha ; \beta)$. Take $M$ to be a model with states $s_{1}, s_{2}, s_{3}$ and let the interpretation of the actions be given by $\tau(\alpha)$ $\left.=\left\{<s_{1}, s_{2}\right\rangle\right\}$ and $\tau(\beta)=\left\{<s_{2}, s_{3}>\right\}$. In $M,<s_{1}, s_{2}>\notin P$ and $<$ $s_{2}, s_{3}>\in P$, so the first transition is not a permitted state transition but the second is. 'Then, $s_{1} \Vdash a \rightarrow \pi \beta$ ( $s_{1}$ supports or accepts the formula $\left.\alpha \rightarrow \pi \beta\right)$ becausc last $\left(s_{1} \llbracket \alpha \rrbracket\right)=s_{2} \Vdash \pi \beta$. The latter holds since $<s_{2}, s_{3}>\in P$, so $\operatorname{Pcrm}\left(<s_{2}, s_{3}>\right)$. On the other hand, $\pi(\alpha ; \beta)$ is not accepted/supported in $s_{1}$ because the sequence $\left\langle s_{1}, s_{2}\right\rangle-\left\langle s_{2}, s_{3}\right\rangle=\left\langle s_{1}, s_{2}, s_{3}\right\rangle$ is not a permitted sequence (the state transition $\left\langle s_{1}, s_{2}\right\rangle$ is not in $\mathrm{P}$ ).

\subsection{The repercussion of actions}

When an imperative expression and a proposition are connected by the connectives and/or, the second conjunct is interpreted as a repercussion of the compliance (47a) or as a repercussion of the failure to comply (47b) with the command in the first conjunct.

(47) a. Go to San Francisco and Jane will be happy 
b. Go to San Francisco or Jane will be unhappy. follows:

The translations of the sentences in (47) into the language of DAL, are as
a. $\square$ Go to San Francisco $\wedge$ Happy $($ Jane $)$
b. $\square$ Go to San Francisco $\vee$ Unhappy(Jane)

The above formulas have as their unique interpretation a "repercussive" one. In other words, according to the update semantics of $\wedge$, the proposition Jane will be happy is interpreted in the state resulting from updating $s$ with the command Go to San Francisco. Similarly, the semantic clause for $\vee$ yields either the set of worlds in which the command is salisfied or the set of worlds resulting from interpreting the proposition Janc will be unhappy in a state in which the addressee is allowed to not going to San Francisco.

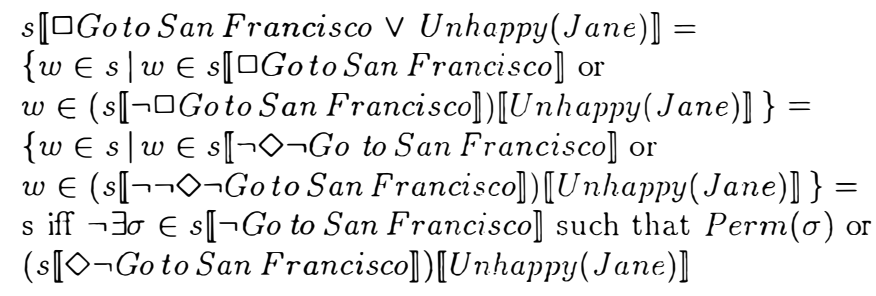

\section{Endnotes}

(*) I would like to thank the audiences at SALT 7 (Stanford, March 1997) and at the 21st Penn Linguistic Colloquium (Upenn, February 1997), especially Nicholas Asher, Hans Kamp, Stanley Peters and Roumyana Izvorski for their • comments.

1. A perhaps more intuitive alternative would be to consider kill(Caesar) as an action expression and relativize it to agents. Here we stick to the simpler option.

2. Let $\tau$ be a temporal trace function mapping a state $s$ to an interval, $\tau(s)=i$, where $i$ is the moment or interval of time occupied by $s$, and let $<$ be an ordering relation between intervals. For any states $s, s^{\prime}$ in a sequence $\sigma$ and such that $\tau(s)=i$ and $\tau\left(s^{\prime}\right)=j, s \prec{ }_{t} s^{\prime}$ iff $i<j$.

3. This is why translating $(40 \mathrm{~b})$ as (i) would be less adequate. The DAL formula in (i) only captures the exclusive weak reading of (40b).

(i) $\diamond(B u y($ an Opel $)($ John $) \sqcup B u y($ a Honda $)($ John $))$

4. Stanley Peters points out that it may be desirable to consider deontic operators as non-monotonic in general and takc inferences related to a.ction extension and decreasingness as the exception. 


\section{References}

Groenendijk, J., Stokhof, M. (1990) "J)ynamic Montague Grammar," in L. Kalman and L. Polos (eds.) Papers Srom the Second Symposium on Lıgic and Language, Budapest, Akadémiai Kaidó.

Groenendijk, J., Stokhof, M. (1991) "Dynamic Predicate Logic," Linguistics and Philosophy 14, 39-100.

Groenendijk, J., Stokhof, M., \& Veltman, F. (1995) "Correference and Modality," in S. Lappin (ed.) Handbook of Semantics, Oxford, Basil Blackwell, 179-213.

Hamblin, C. (1987) Imperatives, Oxford, Basil Blackwell.

Israel, D., Perry, J. and Tutiya, S. (1993) "Executions, Motivations and Accomplishments," The Philosophical Review 102, 515-540.

Kamp, H. (1973) "Free Choice Permission," Proceedings of the Aristotelian Society 74, 57-74.

Kamp, H. (1979) "Semantics versus Pragmatics," in F. Guenthner and S. Schmidt (eds.) Formal Semantics and Pragmatics for Natural Languages, Reidel, Dordrecht, 255-288.

Lewis, D. (1979) "A Problem about Permission," in E. Saarinen et al. (eds.) Essays in Honour of Jaakko Hintikka, Reidel, Dordrecht, 163-175.

Meyer, J. (1988) "A different Approach to Deontic Logic: Deontic Logic Viewed as a Variant of Dynamic Logic," Notre Dame Journal of Formal Logic 29, 109-136.

Pratt, V. (1978) "Process Logic," Proc. 6th ACM Symposium Foundations Comp. Science, ACM Press, New York, 93- 100.

Rohrbaugh, E. (1995) "An Event-Based Semantics for Deontic Utterances," Proceedings of the 10th Amsterdam Colloquium, Amsterdam, ILLC, 635-650.

Ross, A. (1941) "Imperatives and Logic," Theoria $i, 53-71$.

Van der Meyden, R.. (1996) "The Dynamic Logic of Permission," Journal of Logic and Computation 6, 465-479.

Van Eijck, J. and Cepparello, G. (1995) "Iynamic Modal Predicatc Logic," in M. Kanazawa and C. Piñón (eds.) Dynamics, Polarity and Quantification, Stanford, CSLI Publications, 251-276.

Veltman, F. (1996) "Defaults in Update Semantic.s," Journal of Philosophical Logic, 25, 221-261.

Von Wright, G. H. (1969) An Essay on Deontic Logic and the Theory of Action, Amsterdam. 